\title{
Histopathological, Ultrastructural and Apoptotic Changes in Diabetic Rat Placenta
}

\author{
Mehmet Gül ${ }^{1}$, Nuray Bayat ${ }^{1}$, Aslı Çetin ${ }^{1}$, Remziye Aysun Kepekçi², Yavuz Şimşek $^{3}$, Başak Kayhan ${ }^{4}$, \\ Uğur Turhan ${ }^{3}$, Ali Otlu ${ }^{1}$
}

\begin{abstract}
${ }^{1}$ Department of Histology and Embriyology, İnönü University Faculty of Medicine, Malatya, Turkey ${ }^{2}$ Department of Biology, Gaziantep University Faculty of Arts and Science, Gaziantep, Turkey ${ }^{3}$ Department of Obstetrics and Gynecology, İnönü University Faculty of Medicine, Malatya, Turkey ${ }^{4}$ Department of Medical Biology and Genetics, İnönü University Faculty of Medicine, Malatya, Turkey
\end{abstract}

Background: The exchange of substances between mother and fetus via the placenta plays a vital role during development. A number of developmental disorders in the fetus and placenta are observed during diabetic pregnancies. Diabetes, together with placental apoptosis, can lead to developmental and functional disorders. Aims: Histological, ultrastructural and apoptotic changes were investigated in the placenta of streptozotocin (STZ) induced diabetic rats.

Study Design: Animal experimentation.

Methods: In this study, a total of 12 female Wistar Albino rats (control $(n=6)$ and diabetic $(n=6))$ were used. Rats in the diabetic group, following the administration of a single dose of STZ, showed blood glucose levels higher than $200 \mathrm{mg} / \mathrm{dL}$ after 72 hours. When pregnancy was detected after the rats were bred, two pieces of placenta and the fetuses were collected on the $20^{\text {th }}$ day of pregnancy by cesarean incision under ketamine/ xylazine anesthesia from in four rats from the control and diabetic groups. Placenta tissues were processed for light microscopy and transmission electron microscopy (TEM). Hematoxylin-eosin (HE) and periodic acid Schiff-diastase (PAS-D) staining for light microscopic and caspase-3 staining for immunohistochemical investigations were performed for each placenta. Electron microscopy was performed on thin sections contrasted with uranyl acetate and lead nitrate.

Results: Weight gain in the placenta and fetuses of diabetic rats and thinning of the decidual layer, thickening of the hemal membrane, apoptotic bodies, congestion in intervillous spaces, increased PAS-D staining in decidual cells and caspase- 3 immunoreactivity were observed in the diabetic group. After the ultrastructural examination, the apoptotic appearance of the nuclei of trophoblastic cells, edema and intracytoplasmic vacuolization, glycogen accumulation, dilation of the endoplasmic reticulum and myelin figures were observed. In addition, capillary basement membrane thickening, capillary endothelial cells chromatin condensation in the nucleus and corrugation of the nucleus were found. Conclusion: Diabetes causes histomorphometric, ultrastructural and apoptotic changes in rat placenta.

Keywords: Apoptosis, diabetes, histopathology, placenta, ultrastructure 
Diabetic pregnancies are characterized by numerous defects associated with fetal growth and development. Both fetal macrosomia and intrauterine development retardation are frequently seen in uncontrolled diabetic pregnancies (1). Despite currently available treatment, maternal diabetes comprises unfit conditions for embryonic and fetoplacental development. The pregnancies with type 1 or type 2 diabetes have an increased risk of miscarriage, stillbirth, congenital malformations, placental abnormalities, and intrauterine malprogramming (2). However, reports on the pathology of placenta are usually incompatible with diabetes mellitus. The inconsistency may be explained partially by the fact that the category of diabetic pregnant women is not homogeneous. The analysis of placental lesions in maternal diabetes has been complicated by superimposed hypertensive and other associated complications (3). Concerning these crucial risks, the diabetic pregnancies have been widely studied using animal models to determine the risks involved during in utero development.

Streptozotocin (STZ) induced diabetes in rodents is the most well-defined and widely used model system of experimental diabetes. This model is characterized by endogenous chronic oxidative stress, which results from hyperglycemia (4). Exposure to hyperglycemia under the experimental conditions results in reduced weight gain in diabetic rats at the end of pregnancy (4). In STZ induced diabetic rats, the placenta is larger and the fetal weight is higher than in healthy rats; these rats often give birth to macrosomic fetuses $(1,4,5)$. The pathogenesis of these anomalies is not well understood. Verma et al. observed abnormalities in the placenta of both groups of patients whether the blood sugar level was controlled by diet or insulin (3). They indicated that hyperglycemic regulation only partially prevented the development of placental abnormalities. Despite the best glycemic control in the modern management of pregnancy, these malformations still reflect the adverse effects of hyperglycemia on the fetus and the placenta. Altogether, the evidence suggests that other constituent diabetic factors may lead to these abnormalities.

Placental development depends on effective implantation and invasion of the maternal decidua by placental trophoblasts. Apoptosis through the caspase activation in trophoblasts increases in parallel with placental growth and the progression of pregnancy during normal gestation. However, apoptosis is significantly exaggerated in pregnancies complicated by diabetes or pre-eclampsia (6).
Caspases are pivotal mediators of programmed cell death. Among them, caspase-3 has been shown to be a key factor in apoptosis. Caspase- 3 activation can catalyze the specific cleavage of many cellular proteins and irreversibly commit cells to undergo apoptosis, with morphological features such as nuclear condensation and DNA fragmentation. Because caspases are major contributors to the apoptotic machinery in many cell types, selecting potent caspase inhibitors has emerged as a new therapeutic strategy $(7,8)$.

Since caspase-3 is one of the well-known mammalian caspases in terms of its specificity and role in apoptosis (9), we aimed to investigate the histopathological and ultrastructural changes along with caspase-3 immunoreactivity in placentae of the STZ induced diabetic pregnant rats. Increased knowledge of caspase- 3 activation in the diabetic placenta may pave the way for the utilization of selective caspase- 3 inhibitors as novel therapeutics to preserve the fetus and placenta against developmental malformations in pregnancies complicated by diabetes.

\section{MATERIALS AND METHODS}

\section{Experimental design}

Adult female Wistar albino rats were obtained from animal center of İnönü University, Turkey. They were left to acclimate to the animal room for 7 days, and were maintained on a standard pellet diet and water ad libitum at a temperature of $20-25^{\circ} \mathrm{C}$ under a $12 \mathrm{~h} \mathrm{light/dark} \mathrm{cycle} \mathrm{throughout} \mathrm{the} \mathrm{ex-}$ periment. Animals were handled according to the suggested international ethical guidelines for the care of laboratory animals. The Animal Ethics Committee of the İnönü University approved the use of animals for this study (09.06.2011).

The present study was performed on 12 Wistar albino female rats weighing $250-280 \mathrm{~g}$. Six of the rats were chosen randomly and induced with STZ at a dose of $60 \mathrm{mg} / \mathrm{kg}$ of body weight prepared in citrate buffer ( $\mathrm{pH} 7.4$ ), delivered intraperitoneally. After 72 hours, blood samples were taken from tail veins of the rats for glucose determinations following a one-night fasting period. Rats with a blood glucose level of higher than 200 $\mathrm{mg} / \mathrm{dL}$ were regarded as the diabetic group and compared to the control group that were also measured and determined to have normal blood glucose levels (Table 1).

TABLE 1. Maternal blood glucose levels $(\mathrm{mg} / \mathrm{dL})$ of rats in the control and diabetic groups

\begin{tabular}{lllllllll}
\hline Time & C1 & C2 & C3 & C4 & D1 & D2 & D3 & D4 \\
\hline Before gestation & 76 & 84 & 96 & 67 & 98 & 92 & 68 & 72 \\
48 hours after STZ induction & 78 & 84 & 95 & 66 & 280 & 320 & 330 & 301 \\
1. day of gestation & 72 & 80 & 98 & 76 & 256 & 276 & 300 & 260 \\
$20^{\text {th }}$ day of gestation & 95 & 99 & 104 & 97 & 295 & 333 & 326 & 298 \\
\hline
\end{tabular}

\footnotetext{
C: rat in the control group; D: rat in the diabetic group; STZ: streptozotocin
} 
After the induction of diabetes, female rats were mated with non-diabetic male rats in a 1:1 ratio. Vaginal smear samples were taken on the day following mating. The day when spermatozoa were found in the vaginal smear by light microscopy was designated as day 0 of gestation. During the gestation period, maternal weight gain and blood glucose levels were followed regularly in the control and diabetic groups. Four animals became pregnant in each group, so in total eight animals were included in the study.

On day 20 of gestation, the dams were weighed and anesthetized with ketamine/xylazine. Two_placentae from each rat (there were more than one fetus/rat) in both of the groups were removed by caesarean section, weighed, and tissue processing was followed by an appropriate protocol. The ovaries and uterine contents were also examined to determine the number of viable or dead fetuses. The fetuses were weighed upon removal from the placentae.

\section{Histopathological examination}

Placentae were sectioned for histological analysis. For light microscopic evaluation, placenta samples were fixed in phosphate-buffered $10 \%$ formalin. Paraffin-embedded specimens were cut into $5 \mu \mathrm{m}$ thick sections, mounted on slides, and stained with hematoxylin-eosin (H-E), Periodic acid Schiffdiastase (PAS-D), and caspase-3 (rabbit polyclonal antibody to active caspase-3; ab4051, Abcam, UK). Sections of the placenta were examined using a Leica DFC280 light microscope and analyzed using the Leica Q Win Plus V3 Image Analysis System (Leica Micros Imaging Solutions Ltd.; Cambridge, U.K). The thickness of the decidua was measured in ten different areas of placental sections at a 20x magnification.

The H-Score method was used to score the degree of caspase-3 positive immunoreactivity of the placenta tissues. This immunohistochemical semi-quantitative method consists of the percentages of positively stained cells multiplied by the intensity of staining: $\mathrm{H}$ Score $=\mathrm{Pi}(\mathrm{i}+1)$, where $\mathrm{Pi}$ is the percentage of stained cells in each intensity category $(0-100 \%)$ and $i$ is the intensity indicating weak $(i=1)$, moderate $(i=2)$ or strong staining $(\mathrm{i}=3)$. Caspase- 3 immunoreactivity was statistically evaluated according to the $\mathrm{H}$-score and compared with each other.

For electron transmission microscopic examination, samples of the placenta were fixed in $2.5 \%$ glutaraldehyde (Merck, Germany) buffered with $0.2 \mathrm{M} \mathrm{NaH} 2 \mathrm{PO} 4+\mathrm{NaHPO} 4(\mathrm{pH}$ 7.4 ) and post-fixed in $1 \%$ osmium tetroxide (OsO4) (Merck, Germany). After dehydration in acetone, tissues were embedded in Araldite CY 212. Ultrathin sections were stained with uranyl acetate and lead citrate and examined using a Zeiss Libra 120 transmission electron microscope (Carl Zeiss NTS GmBH, Oberkochen, Germany).
TABLE 2. Mean values of maternal blood glucose levels, fetal birth weight, and placental weight in the control and diabetic groups

\begin{tabular}{lcc}
\hline & Control group & Diabetic group \\
\hline Blood glucose $(\mathrm{mg} / \mathrm{dL})$ & 89 & $275^{*}$ \\
Birth weight of fetus $(\mathrm{g})$ & $4.1 \pm 1.6$ & $5.1 \pm 0.7^{*}$ \\
Placental weight $(\mathrm{g})$ & $0.7 \pm 0.1$ & $0.9 \pm 0.2^{*}$ \\
\hline
\end{tabular}

*indicates $\mathrm{p}<0.05$ compared with the control group

TABLE 3. H scores of caspase- 3 immunoreactivity in the control and diabetic groups

\begin{tabular}{lc}
\hline Group & Mean \pm SD \\
\hline Control & $0.5 \pm 0.93$ \\
Diabetic & $68.8 \pm 12.5^{* *}$ \\
\hline
\end{tabular}

$* *$ indicates $\mathrm{p}<0.005$ compared with the control group

\section{Statistical analysis}

All data were statistically analyzed by using SPSS 13.0 (SPSS inc., Chicago, IL, USA) software and expressed as mean \pm standard deviation. Normality for continuous variables in groups was determined by the Kolmogorov - Smirnov test. Comparison of continuous variables among groups was performed by Mann - Whitney U test variables with non-normal distribution. $\mathrm{p}<0.05$ was regarded as significant.

\section{RESULTS}

\section{Effects on dams, fetuses and placenta}

The blood glucose levels of rats in the diabetic group were higher than the rats in the control group during and at the end of the experiment (Table 1). The weights of the placentae and the fetuses in the diabetic group were higher than those in the control group (Table 2). The maternal weight gain during pregnancy in the diabetic group (123 g) was higher than in the control group $(57 \mathrm{~g})(\mathrm{p}<0.05)$.

At the end of gestation, a reduction in the number of living fetuses was detected in the diabetic group in comparison to the control group. Based on this, we obtained 6-8 living fetuses and 4-5 dead fetus from each rat in the diabetic group and 10-12 living fetuses and 6-8 dead fetuses from each rat in the control group by caesarean section.

\section{Histopathological results}

All placental sections of the control group were normal in histological structure according to light microscopic analysis. Intervillous spaces were open (Figure 1a) and the basal laminae of the hemal membrane was PAS-D+ (Figure 1b). Caspase-3 immunoreactivity was at a minimal level (Table 3) in these sections (Figure 1c). The trophoblastic cells 

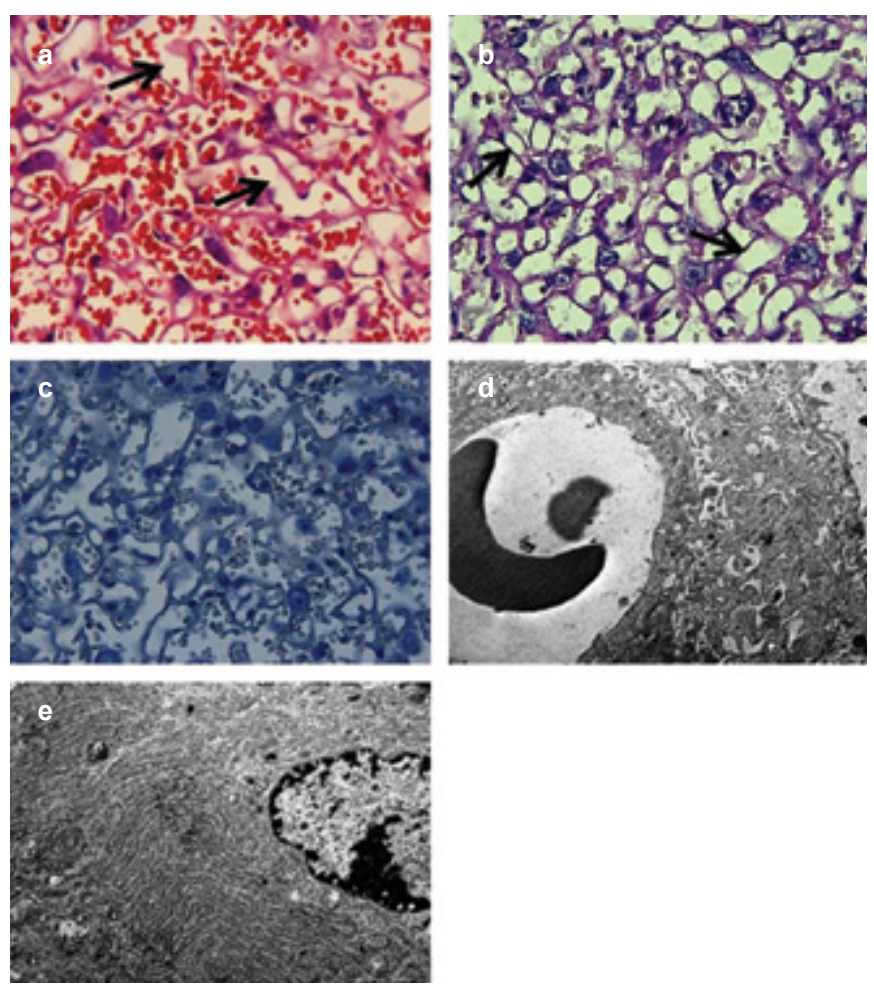

FIG. 1. a-e. The histological structure of the placenta in the control group as observed by light microscopy (a, b, c) and electron microscopy (d, e). In the control group, the placenta had a normal histological appearance; the intervillous range was explicit (H-E; 20x) (a), hemal membranes were thin (arrow) and the basement membrane in the hemal membranes showed PAS-D positivity (arrow) (PAS-D; 40x) (b), whereas no positivity was observed for caspase-3 staining. Ccaspase-3; 40x. (c). The trophoblastic cells and vascular structures were observed as in the normal ultrastructure $(d, e)$; scale bar $=2 \mu \mathrm{m}$.

and the vascular structures were evaluated as ultrastructurally normal according to electron microscopic analysis in this group (Figure 1d, e).

Apoptotic corpuscles were observed in the maternal and fetal areas and intensive congestion in intervillous spaces in the H-E stained placental sections of the diabetic group. Decidual cells were heavily PAS-D + stained in maternal placenta areas. Additionally, significant thickening of the hemal membranes were detected in these cells (Figure 2a-d). The H-score of caspase-3 immunoreactivity was determined to be higher in the diabetic group compared to the control group $(p<0.005)$ (Table 3). The thickness of the decidual membrane (440.60 \pm 85.69$)$ was thinner than in the control group (689.46 \pm 142.9$)$ (Table 4).

Electron microscopic analysis of the placenta in the diabetic group also showed the following findings: apoptotic nuclei, intracytoplasmic vacuolization, intracellular edema, lipid droplets, increased lysosomes, glycogen accumulation, mitochondrial injury and degeneration, dilatation of the endoplasmic reticulum, myelin figure formation in trophoblast
TABLE 4. Thickness $(\mu \mathrm{m})$ of the decidual layer in the control and diabetic groups

\begin{tabular}{lc}
\hline Group & Mean \pm SD \\
\hline Control & $689.46 \pm 142.9$ \\
Diabetic & $440.60 \pm 85.7 * *$ \\
\hline$* *$ indicates $p<0.005$ compared with the control group
\end{tabular}

cells, thickening in the capillary basal membrane, peripheral chromatin condensation in the capillary endothelial cell nucleus, and undulations in the boundaries of the nucleus. Besides these results, an increase in intercellular distance was also evident (Figure 2f-m).

\section{DISCUSSION}

Normal fetal growth and development largely depended on the function of the placenta. Some forms of intrauterine fetal growth retardations have been correlated with insufficient placental blood flow and transfer of nutrients from mother to fetus in humans as well as in animals (10).

The physiological changes that occur during pregnancy can only be sustained if there is an appropriate nutrient supply to ensure placental and fetal development (11). To make sure the supply of nutrients essential to fetal survival, the placenta continuously undergoes changes in weight, structure, shape, and function during gestation (12). The most frequently observed abnormality in human placentae is the placental enlargement, in which leads to neonatal macrosomia. The increased glucose transfer and growth promoting effects of insulin among other hormones have been suggested as important mediators of these abnormalities (1).

In the present study, we detected larger placentas and macrosomic living fetuses in diabetic rats with respect to non-diabetic controls. This placental enlargement was regarded as a compensatory mechanism, which is needed to provide enough nutrient and oxygen to fetuses under limited oxygen supply (1). Despite placentomegaly, decreases in placental blood flow in parallel with fetal growth retardation have been previously reported as a result of increased erythropoietin and secondary polycythemia caused by hypoxia in the fetus. A similar pattern of fetal growth retardation caused by decreased placental blood flow has been correlated with placentomegaly in pregnant women living at high altitudes and using alcohol (6). In this study, histologically distinctive differences were detected in the placenta of diabetic rats with respect to rats in the control group. The placentae of diabetic rats displayed significant cell hyperplasia and thickening of the basal membrane of syncytial cells, while these could not be observed in the placentae of rats in the control group. Cytotrophoblastic cell prolifera- 

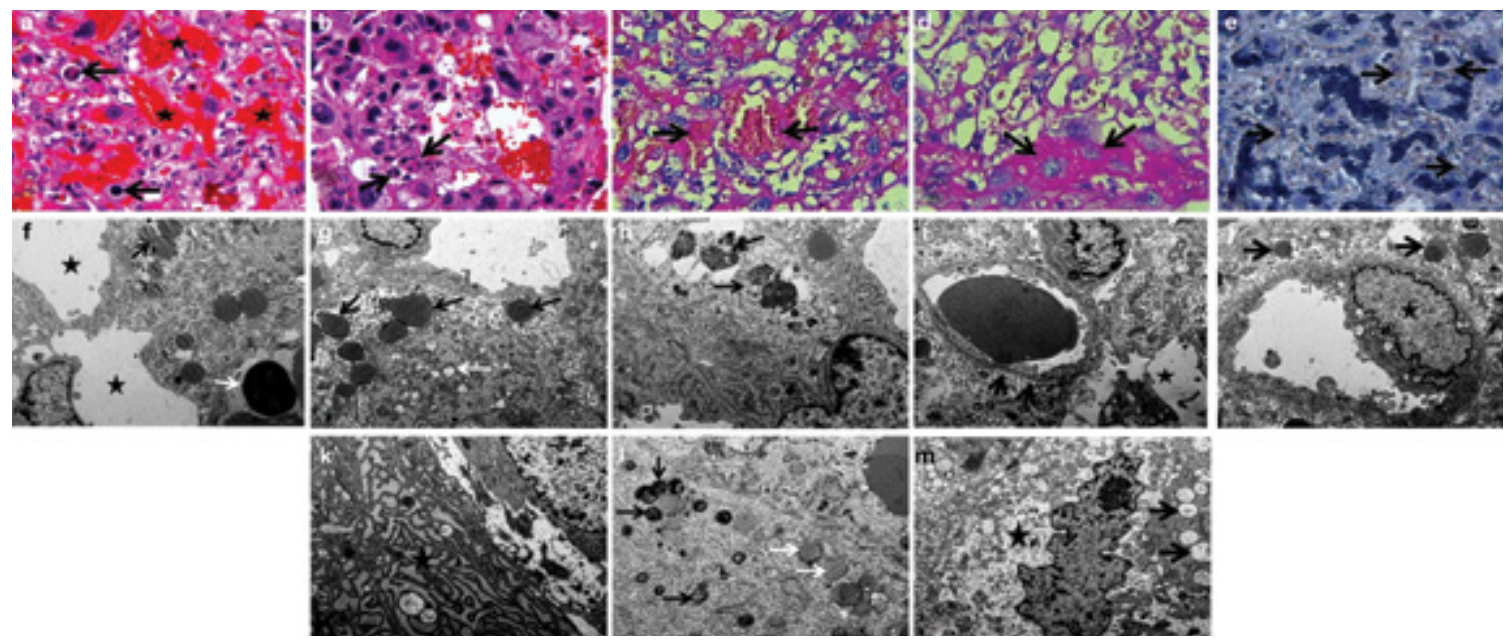

FIG. 2. a-m. The histopathological findings of the placenta in the diabetic group observed by light microscopy (a, b, c, d, e) and electron microscopy (f, g, h, i, j, k, I, m).

Apoptotic bodies (arrow) were observed in fetal areas of the placenta, $(\mathrm{H}-\mathrm{E} ; 40 \mathrm{x})$. (a) Intensive congestion (asterisk) in intervillous spaces (HE; X40). (b) Significant thickening in hemal membranes (arrow). H-E; 40x. (c) Decidual cells stained as PAS-D + in maternal placenta areas PAS-D; 40x). (d) Positive caspase-3 immunoreactivity (caspase-3; 40x) (e) was observed in the placentae of diabetic rats by light microscopy. Electron microscopic analysis of diabetic placentae revealed the following findings: apoptotic nucleus structure (white arrow) in trophoblast cells, the distinctive extension in intercellular distances (asterisk), (f) lipid droplets (black arrow) (f, l), increase in lysosomes (black arrow) ( $\mathrm{g}, \mathrm{h}$, $\mathrm{j})$, mitochondrial injury and degeneration (white arrow) (g), (black arrow) $\mathrm{m}$ ), dilatation of the endoplasmic reticulum (asterisk) (k), myelin figure formation (black arrow) (I), intracytoplasmic vacuolization and intracellular edema (asterisk) (m). Furthermore, thickening in the capillary basal membrane (black arrow) (i), peripheral chromatin condensation in the capillary endothelial cell nucleus and undulations in the boundaries of the nucleus (asterisk) (j) were also detected (f, g, h, i, j, k, l, m); scale bar=2 $\mu \mathrm{m}$.

tion is accepted as an indicator of uteroplacental ischemia, as reported in other studies (10). In this study, we observed an increase in embryonic loss, a decrease in the number of living fetuses, and a lower rate of implantation in diabetic rats. Supporting our results, Sinzato et al. (6) also demonstrated that diabetic rats had decreased numbers of living fetuses, implantations and corpora lutea, and an increased rate of embryonic loss. They reported that diabetes triggered alterations in the maternal organism, leading to impaired decidua development contributing to failure in embryonic implantation and early embryonic losses. We determined marked thinning in the decidual area in diabetic placentae, compared to the control ones. Decidualization, characterized by the differentiation of the uterine stromal cells, is an adaptation of uterus related to gestation (13). Differentiation of decidual cells is dependent on the regulatory effect of ovarian steroids such as progesterone and $17 \beta$-estradiol. These cells are responsible for the control of changes in the maternal and extra-embryonic tissues to provide for the permanence of gestation (14).

It has been suggested that increased glycaemia prior to pregnancy (or its increase in early pregnancy) might be linked to a reduction in the number of corpora lutea in diabetic animals (1). A reduction in the decidual area and the number of corpora lutea might be related to the decline in the secretion of progesterone (6). During oocyte development, the surround- ing granulosa cells support oocyte development and provide hormonal supplementation. The hyperglycemic state affects the intercellular communication of granulosa cells, and results in poor paracrine communication leading to oocyte maturation delay (15). This phenomenon could explain the reduced rate of implantation in the diabetes model.

Exposure to hyperglycemia resulted in elevated maternal weight gain and elevated fetus weight in the diabetic dams at the end of pregnancy under the experimental conditions. The mechanisms underlying may involve destruction of the mother's pancreas and lipid abnormalities together with oxidative stress at birth $(16,17)$. The other finding we detected in our study was distinct thickening of the interhemal membranes of trophoblastic cells in diabetic placentae. This was also previously shown to be more explicit in the uncontrolled diabetic placenta (18). This basal membrane thickening could be the result of mucopolysaccharide storage (19), which results in maternal uteroplacental vascular insufficiency and intrauterine growth retardation (1). In addition, we also observed thickening of the capillary basal membrane of diabetic rat placentae, which leads to an increased diffusion distance between maternal and fetal blood (10).

The findings from our study reveal distinctive changes in diabetic rat placentae with respect to those of control rats, including thickening of the basal membrane of trophoblastic 
cells, villous edema, congestion in intervillous spaces, and a decrease in the thickness of the decidual membrane. These differences are accepted as indicators of disruption of placental exchange, which affects the rate of implantation, the number of living fetus, and fetal and placental weight (6). Electron micrographs have also revealed giant cells filled with glycogen in the basal zone of the diabetic placenta, which may also disrupt blood flow and placental exchange by increasing the transfer distance from maternal blood (1). This glycogen accumulation in the placenta was probably caused by high levels of glucose supplied by the maternal blood circulation in diabetic rats as well in diabetic women. The presence of maternal hyperglycemia in late pregnancy causes insulin levels in the fetal circulation to increase. Thus, the maternal side of the placenta is exposed to norm-/hypoinsulinemia while the fetal side is subject to hyperinsulinemia (19). In accordance with this data, the accumulation of lipid droplets in the cytoplasm of trophoblastic cells could be explained by fetal hyperinsulinemia (1). Compared with the control group, the other prominent changes we detected in the placentae of diabetic rats were also in accordance with the findings of previous studies. These changes included an increase in the thickness of the vasculosyncytial membrane (20), trophoblastic hyperplasia (3), villous edema, glycogen accumulation in all layers of the placenta, dilation of the endoplasmic reticulum in varying degrees and mitochondrial degeneration (21). These changes are regarded as pivotal factors contributing to fetal anoxia in gestation complicated by diabetes (20). Hyperglycemia-induced abnormalities in placental metabolism have been postulated to contribute to the development of malformations in embryonic cells (3). A considerable amount of clinical and experimental evidence now exists suggesting the involvement of free radical-mediated oxidative processes in the pathogenesis of diabetic complications (22). Oxidative stress can result in increased apoptosis by damaging other macromolecules vital to cellular functions, signal transduction and genomic processes (23).

In the present study, we frequently observed myelin figure formation, which represents irreversible cell damage in the cytoplasm of trophoblast cells in the diabetic rat placenta. Myelin figures are classically derived from the membranes of cytoplasmic organelles. They are in phospholipid structure and can be seen intracellularly. They are formed from cytoplasmic membranes, and are localized as extracellular and seen as fibrillar eosinophilic deposits and indicate "irreversible" cell damage (24).

Other findings we detected in the diabetic group were dilation of the endoplasmic reticulum and degeneration of mitochondria in varying degrees. These observations support the findings of other studies regarding these defects as indicators of oxidative stress induced cell injury in diabetes (25). Re- cently, it has been hypothesized that hyperglycemia-induced injury of the membranes of embryonic cells is associated with the disruption of cellular signal transduction pathways, especially signal-mediated apoptotic pathways (26).

Among caspases, caspase- 3 is considered one of the predominant targets involved in reactive oxygen species-mediated high glucose-induced apoptosis (27). Supporting this data, we observed an increase in caspase-3 immunoreactivity as an indicator of apoptosis in maternal and fetal areas of diabetic placentae. We found that the H-scores of the caspase- 3 immunoreactivity in diabetic placentae were significantly higher $(66.3 \pm 5.64)$ than those $(0.5 \pm 0.33)$ in the control placentae (Table 3). It seems likely that agents that block apoptotic pathways may be of value for treating diabetes in pregnancy to prevent embryonic malformation. New studies can be planned to assess the function of caspase- 3 inhibitors.

In conclusion, diabetes induces embryonic malformation during pregnancy that is caused, not only by an elevation in caspase-3 levels in maternal and fetal areas of placenta, but also by irreversible cell damage in trophoblast cells of the placenta according to ultrastructural analysis.

Ethics Committee Approval: Ethics committee approval was received for this study from the ethics committee of İnönü University Medicine Faculty Experimental Animasl Ethics Committee (2011/A-59).

\section{Informed Consent: N/A.}

Peer-review: Externally peer-reviewed.

Author contributions: Concept - M.G., Y.Ş., U.T, B.K.; Design - M.G., Y.Ş., N.B., B.K., U.T., A.Ç.; Supervision - M.G., Y.Ş., B.K., A.O.; Resource - M.G., N.B., Y.Ş.; Materials - M.G., Y.Ş., U.T.; Data Collection \&/or Processing - M.G., B.K., Y.Ş.; Analysis \&/or Interpretation - M.G., N.B., B.K.; Literature Search - M.G., N.B., B.K.; Writing - M.G., B.K., R.A.K.; Critical Reviews - M.G., B.K., N.B.

Acknowledgements: The authors thank to Dr. Yusuf Türköz for his supports.

Conflict of Interest: No conflict of interest was declared by the authors.

Financial Disclosure: The authors declared that this study has received financial support from İnönü University Research Project (BAP 2011/168).

\section{REFERENCES}

1. Gewolb I, Merdian W, Warshaw J, Enders A. Fine structural abnormalities of the placenta in diabetic rats. Diabetes 1986;35:1254-61. [CrossRef] 
2. Higgins M, Felle P, Mooney E, Bannigan J, McAuliffe F. Stereology of the placenta in type 1 and type 2 diabetes. Placenta 2011;32:564-9. [CrossRef]

3. Mathiesen ER, Ringholm L, Damm P. Stillbirth in diabetic pregnancies. Best Pract Res Clin Obstet Gynaecol 2011;25:105-11. [CrossRef]

4. Greene DA, Stevens MJ, Obrosova I, Feldman EL. Glucoseinduced oxidative stress and programmed cell death in diabetic neuropathy. Eur J Pharmacol 1999;375:217-23. [CrossRef]

5. Vambergue A, Fajardy I. Consequences of gestational and pregestational diabetes on placental function and birth weight. World J Diabetes 2011;2:196-203.

6. Sinzato YK, Volpato GT, Iessi IL, Bueno A, Calderon IdMP, Rudge MVC, et al. Neonatally Induced Mild Diabetes in Rats and Its Effect on Maternal, Placental, and Fetal Parameters. Exp Diabetes Res 2012;2012:108163. [CrossRef]

7. Sharp AN, Heazell AE, Crocker IP, Mor G. Placental apoptosis in health and disease. Am J Reprod Immunol 2010;64:159-69. [CrossRef]

8. Ferrer I, Planas AM. Signaling of cell death and cell survival following focal cerebral ischemia: life and death struggle in the penumbra. J Neuropathol Exp Neurol 2003;62:329-39.

9. Porter AG, Jänicke RU. Emerging roles of caspase-3 in apoptosis. Cell Death Differ 1999;6:99-104. [CrossRef]

10. Honda M, Toyoda C, Nakabayashi M, Omori Y. Quantitative investigations of placental terminal villi in maternal diabetes mellitus by scanning and transmission electron microscopy. Tohoku J Exp Med 1992;167:247-57. [CrossRef]

11. Gluckman PD, Harding JE. Nutritional and hormonal regulation of foetal growth-involving concepts. Acta Paediatr Suppl 1994;399:60-3. [CrossRef]

12. Toledo M. T, Ventrucci G, Marcondes M. C. Cancer during pregnancy alters the activity of rat placenta and enhances the expression of cleaved PARP, cytochrome-c and caspase 3. BMC Cancer 2006;6:168. [CrossRef]

13. Aplin J. Maternal influences on placental development. Sem Cell Dev Biol 2000;115-25.

14. Brosens J, Gellersen B. Death or survival-progesterone-dependent cell fate decisions in the human endometrial stroma. $J$ Mol Endocrinol 2006;36:389-98. [CrossRef]
15. Chang AS, Dale AN, Moley KH. Maternal diabetes adversely affects preovulatory oocyte maturation, development, and granulosa cell apoptosis. Endocrinology 2005;146:2445-53. [CrossRef]

16. Gheorman L, Pleşea I, Gheorman V. Histopathological considerations of placenta in pregnancy with diabetes. Rom J Morphol Embryol 2012;53:329-36.

17. Schaffer SW, Mozaffari MS. The neonatal STZ model of diabetes. In: McNeill JH, editor. Experimental models of dia- betes. Boca Raton, FL: CRC Press, 1999:231-55.

18. Soulimane-Mokhtari NA, Guermouche B, Yessoufou A, Saker M, Moutairou K, Hichami A, et al. Modulation of lipid metabolism by n-3 polyunsaturated fatty acids in gestational diabetic rats and their macrosomic offspring. Clin Sci (Lond) 2005;109:287-95. [CrossRef]

19. Benirschke K, Kaufmann P, Baergen RN. 6th ed. Berlin-Germany: Pathology of the human placenta; 2006.

20. Barash V, Gutman A., Shafrir E. Fetal diabetes in rats and its effect on placental glycogen. Diabetologia 1985;28:244-9. [CrossRef]

21. Pietryga M, Biczysko W, Wender-Ozegowska E, Brazert J, Biegańska E, Biczysko R. Ultrastructural examination of the placenta in pregnancy complicated by diabetes mellitus. Ginekol Pol 2004;75:111-8.

22. Jones C, Fox H. Placental changes in gestational diabetes: an ultrastructural study. Obstet Gynecol 1976;48:274-80.

23. Damasceno DC, Volpato GT, Paranhos C, I. D. M., Cunha R. Oxidative stress and diabetes in pregnant rats. Anim Reprod Sci 2002;72:235-44. [CrossRef]

24. Kannan K, Jain SK. Oxidative stress and apoptosis. Pathophysiaology 2000;7:153-63. [CrossRef]

25. Majno G, Joris I. Cells, Tissues, and Disease: Principles of General Pathology: Oxford University Press, USA 2004; 1005.

26. Giacco F, Brownlee M. Oxidative stress and diabetic complications. Circ Res 2010;107:1058-70. [CrossRef]

27. Reece EA, Ma XD, Zhao Z, Wu YK, Dhanasekaran D. Aberrant patterns of cellular communication in diabetes-induced embryopathy in rats: II, apoptotic pathways. Am J Obstet Gynecol 2005;192:967-72. [CrossRef]

28. Ho FM, Liu SH, Liau, CS, Huang PJ, Lin-Shiau SY. High glucose-induced apoptosis in human endothelial cells is mediated by sequential activations of c-Jun $\mathrm{NH}(2)$-terminal kinase and caspase-3. Circulation 2000;101:2618-24. [CrossRef] 